\title{
Laser Photochemistry and Hole Burning Spectroscopy in Polymers and Glasses: External Field Effects
}

\author{
L. KADOR, R. PERSONOV,* W. Richter, \\ Th. SESSELMANN, and D. HAARER \\ Physikalisches Institut and BIMF, Universität Bayreuth, \\ Postfach 101251, D-8580 Bayreuth, FRG \\ *Institute of Spectroscopy, USSR Academy of Sciences, \\ 142092 Moscow Region, Troitzk, USSR
}

(Received August 11, 1986)

\begin{abstract}
Photochemical hole burning is a spectroscopic method based on site-selection via laser irradiation. It can be shown, that it yields a $10^{3}$ to $10^{4}$-fold increase in resolution if one deals with amorphous materials such as solid polymers. For various doped polymers (polyethylene, polystyrene, poly(methyl methacrylate), and poly(vinyl butyral)) we have made experiments under low electric field and strain field conditions. These experiments yield both, matrix parameters such as compressibilities and molecular parameters such as molecular dipole moment changes and polarizability changes. We will document, that the amorphous nature of the matrix breaks the molecular symmetry of the dopant molecules and gives rise to internal electric dipole moments or internal fields, which are absent in a periodic crystalline structure.

KEY WORDS Hole Burning / Pressure Tuning / Solvent Shift /

Compressibilities / Stark Effect / Dipole Moments / Internal Fields /
\end{abstract}

In the past the optical spectroscopy of amorphous polymers and glasses had been severely limited by the large bandwidths which are characteristic of disordered media. The method of photochemical hole burning, which had been developed in the past ten years ${ }^{1,2}$ has brought about a $10^{3}$ to $10^{4}$-fold increase in spectral resolution and, hence, has made it feasible to study subtle matrix effects, which shed light on important molecule-matrix interactions.

The basic principle of the hole burning method has been described by various review articles; $;^{3-5}$ therefore we only briefly summarize some of the salient features of the method:

a) The method uses doped samples with concentrations of guest molecules of about $10^{-4}-10^{-6} \mathrm{~mol} \mathrm{~mol}^{-1}$. These dopant molecules are investigated spectroscopically as molecular probes yielding information on in- teraction potentials and interaction fields.

b) The high resolution of the method is achieved by site-selective laser chemistry at low temperatures.

c) The resolution is limited by the 'quasihomogeneous' linewidth of the hole burning spectra. Typical linewidths are $10^{-4}$ to $1 \mathrm{~cm}^{-1}$ at temperatures between 0.3 and $20 \mathrm{~K}$. At higher temperatures $(\geqq 20 \mathrm{~K})$ the resolution gain of the method becomes marginal.

In this paper the hole burning method is described as a new and sensitive means of studying external field effects. These field effects shed light on various molecular parameters such as:

a) molecular interaction potentials

b) small molecular dipole and polarizability changes

c) internal electric fields and strain fields.

All of the above parameters reflect mole- 


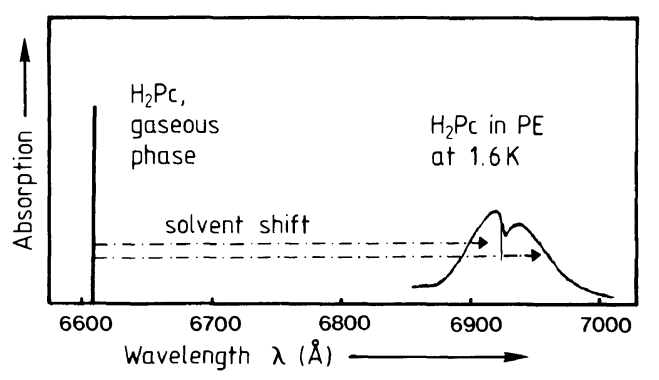

Figure 1. Optical absorption of the dye molecule $\mathrm{H}_{2} \mathrm{Pc}$ in the gasphase as compared to its absorption in solid solution at low temperatures (see the text).

cular interactions, yet they are intimately related to the macroscopic properties of the amorphous materials like for instance the compressibility $\kappa$ of the material or the critical electrical field strengths which determine electrical breakdown phenomena.

The paper will also point towards a relation between the above discussed molecular interaction parameters and the widths of the inhomogeneous bands; it will be shown, that the latter reflect the spread of molecular fields and interactions as is brought about by the amorphous nature of the matrix.

To put the various shift and linewidth parameters into perspective, Figure 1 shows the optical absorption of the dye molecule free base phthalocyanine $\left(\mathrm{H}_{2} \mathrm{Pc}\right)$ in the gasphase, as compared to its absorption in a partially amorphous host material, namely polyethylene (PE). The width of the inhomogeneous band reflects the spread of molecular interaction energies. A photochemical hole, which is burned into the center of the inhomogeneous band gives a measure of the resolution of the involved laser method. In our present paper the focus will always be on the narrow zerophonon part of the hole burning spectrum (narrow dip in Figure 1); the broad phonon satellite (pseudo phonon band) at the long wavelength side will not be further discussed (see for instance ref 5).

\section{HYDROSTATIC PRESSURE EFFECTS}

As has been pointed out in the introduction both, the solvent shift and the width of the inhomogeneous band (Figure 1) reflect the dye-matrix-interaction potential. ${ }^{6}$ The latter is given by a steep, repulsive part and by a more slowly varying attractive part. Solvent shifts have recently been calculated in great detail (see ref 7 and references therein). In the particular case of nonpolar dye molecules in nonpolar solvents, i.e., for purely dispersive interactions and for simple spherical geometries the solvent shift follows a radial dependency as has been derived by Longuet-Higgins and Pople some 20 years ago ${ }^{8}$

$$
\Delta \bar{v}_{0}=-\frac{1}{6 h c} \alpha_{\mathrm{B}} z \frac{1}{\bar{R}^{6}}\left(\frac{1}{4} \varepsilon_{i} \alpha_{\mathrm{A}}+M_{0 \rightarrow i}{ }^{2}\right)
$$

$\alpha_{B}$ and $\alpha_{A}$ are the polarizabilities of the solvent and the solute, respectively, $z$ is the number of the nearest neighbours in the first coordination shell, $\varepsilon_{i}$ is the average energy of the excited state, $\boldsymbol{M}_{0 \rightarrow i}$ is the transition dipole and $\bar{R}$ is the mean distance to the nearest neighbours. Equation 1 assumes that the dielectric medium, surrounding the probe molecule, can be described by a 'solvent cage.' This assumption eliminates the necessity of integrating over the sample volume.

Under uniaxial or hydrostatic pressure the radius of the above defined solvent shell is modified, leading to a change in the solvent shift. This is known from high pressure experiments, in which the whole inhomogeneous band is shifted to lower or higher energies. ${ }^{9-11}$ Since the typical inhomogeneous bandwidths are large, the experimental pressures which are required are on the order of $1 \mathrm{GPa}(10 \mathrm{kbar})$. These large external pressures must be considered as sizeable perturbations on the equilibrium potential. They are almost comparable to a quantity which we could call 'internal pressure.' The latter can be defined as the pressure, which one would have to apply in order to achieve a lineshift comparable to the 
solvent shift.

Since the method of photochemical hole burning leads to a $10^{3}$ to $10^{4}$-fold increase in spectral resolution, one can investigate pressure shifts which are correspondingly smaller. This means, that we can operate with our experiments close to the equilibrium positions of the involved molecular potentials and, hence, obtain matrix parameters (for instance compressibilities) which are rather close to the values which are mechanically measured under equilibrium conditions.

\section{HYDROSTATIC PRESSURE EXPERIMENTS}

In our pressure experiments, we examined poly(methyl methacrylate) (PMMA), polystyrene (PS), and polyethylene (PE). They were doped with the dye molecule free base phthalocyanine $\left(\mathrm{H}_{2} \mathrm{Pc}\right)$ (for the structural formula see Figure 2a). The polymers were doped with dye concentrations of about $10^{-4} \mathrm{~mol} \mathrm{~mol}^{-1}$. The temperature of the experiments was $1.6 \mathrm{~K}$. Both the hole burning photochemistry and the subsequent spectroscopy were performed with a stabilized tunable dye laser with a resolution of about $3 \mathrm{MHz}$. The investigated optical transition was the $S_{1} \leftarrow S_{0}$ transition of the dye molecule (for details see ref 5 and references therein). Only the zero phonon part of the hole burning spectrum was investigated. External strain fields, i.e., both uniaxial and hydrostatic pressures were applied. In the case of uniaxial pressure, ${ }^{12}$ however, the sensitivity of the method is so large, that small irreversible sample deformations lead to irreversible line broadenings, which show up under the high resolution conditions. The reason for the observed irreversible line broadenings is, that it is close to impossible to have ideal plane-parallel samples and to have a pressurizing setup which exerts the uniaxial pressure in a completely homogeneous fashion. Therefore a slightly wedgeshaped sample arrangement will lead to irreversible strains which will prevent the sample from returning to its 'virgin state' after a pressure cycle has been performed.

In this paper we report hydrostatic hole burning experiments up to a pressure of $20 \mathrm{MPa}$ for investigating our samples. The experiments were performed with liquid $\mathrm{He}$ as pressure transmitting medium in a special pressure cell (to be published elsewhere). Due to the high resolution of our method of several hundred $\mathrm{MHz}$ the measured pressure shifts of up to $10 \mathrm{GHz}$ are sufficient to perform a very accurate analysis of the lineshifts. In fact, pressure variations of as little as $100 \mathrm{~h} \mathrm{~Pa}$ are sufficient to produce measurable changes in

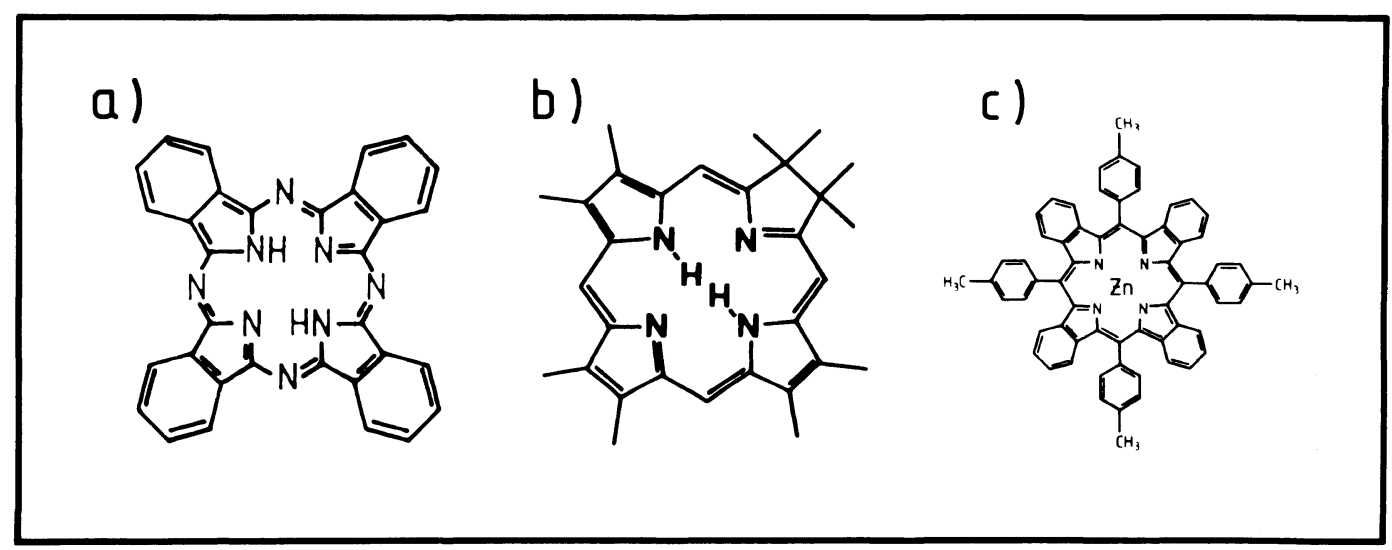

Figure 2. Structures of the dye molecules used in this hole-burning study: free-base phthalocyanine (a), free-base chlorin (b) and $\mathrm{Zn}-\mathrm{TBP}\left(\mathrm{C}_{6} \mathrm{H}_{4} \mathrm{CH}_{3}\right)_{4}$ (c). 


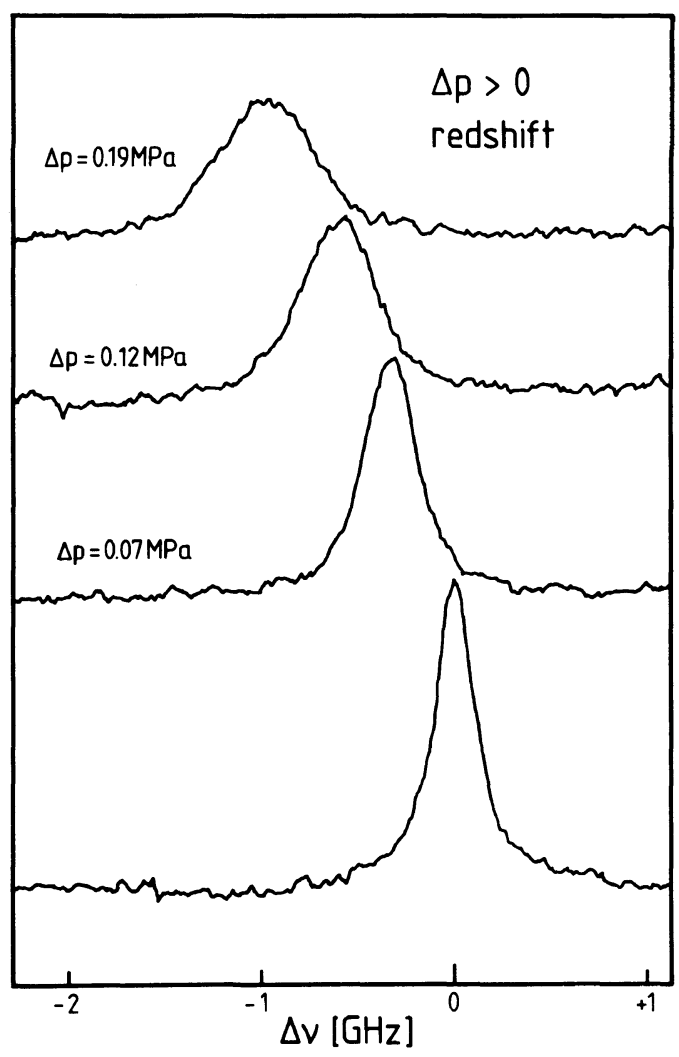

Figure 3a. Typical redshift of the spectral hole with increasing pressure $\left(\mathrm{H}_{2} \mathrm{Pc}\right.$ in $\left.\mathrm{PE}\right)$.

the hole spectra. These experimental figures have to be compared to typical pressures required to shift measurably 'broad line spectra' which are on the order of several tens of GPa. ${ }^{9-11}$

In our hydrostatic low pressure regime all measured changes in linewidth and lineshape turned out to be completely reversible. The pre-pressure, i.e., the pressure under which the hole was produced via site selection photochemistry did not influence the measured linewidths within our experimental accuracy. Therefore only the pressure difference $\Delta p$ enters into our experiments; this means that we can also investigate the influence of 'negative pressure' by burning our hole at high pressure and then reducing the external pressure.

Figures $3 a$ and $b$ show typical lineshifts and

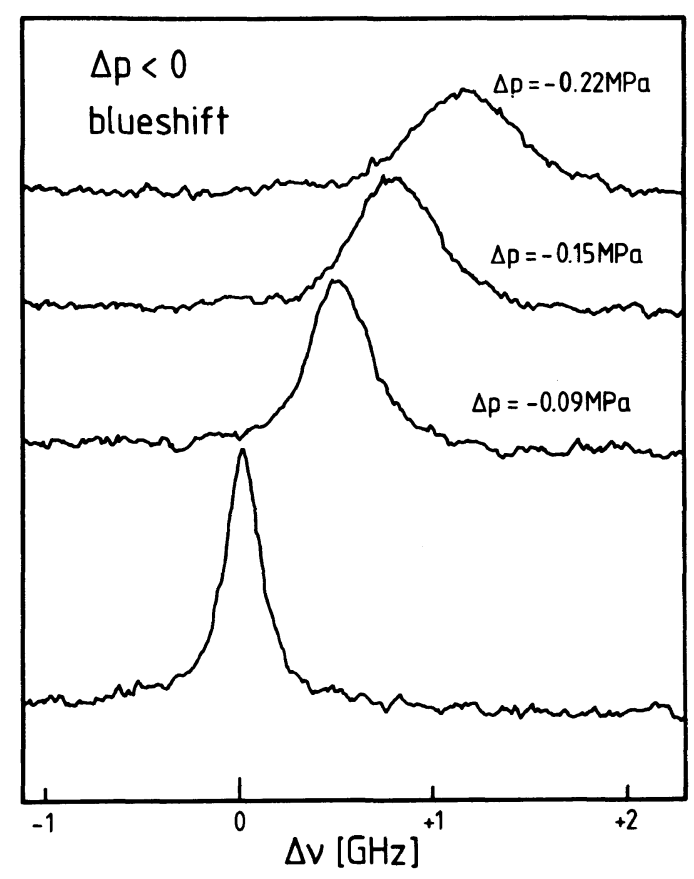

Figure 3b. Typical blueshift of the spectral hole with decreasing pressure $\left(\mathrm{H}_{2} \mathrm{Pc}\right.$ in $\left.\mathrm{PE}\right)$.

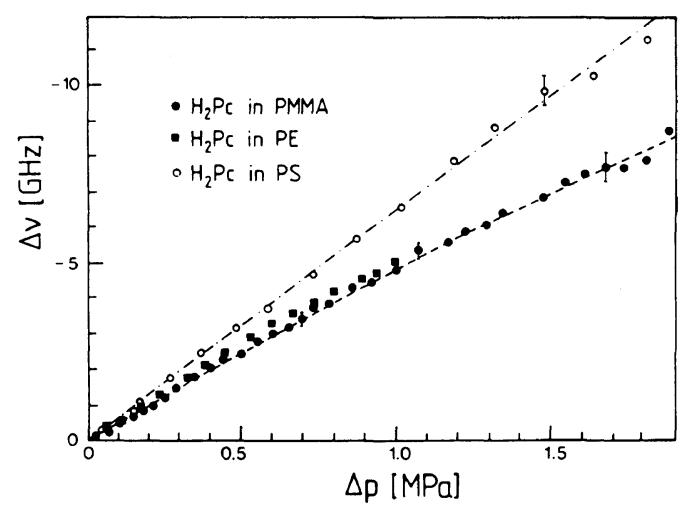

Figure 4. Spectral shifts as a function of increasing pressure.

broadenings for pressure variations of several hundred $\mathrm{hPa}$, i.e., pressure shifts which would not show up in a straight forward optical experiment.

Figure 4 shows the measured red shifts $\Delta \bar{v}(p)$ of the hole spectra for $\mathrm{H}_{2} \mathrm{Pc}$ in PMMA, PS, and PE. Note, that the linear shift term is the dominant contribution to our observed 
Table I. Pressure shifts $\Delta \bar{v}(p) / \Delta p$ and pressure broadenings $\Delta \gamma(p) / \Delta p$ of the holes with increasing pressure for the different matrices $\mathrm{PE}$, PS, and PMMA

\begin{tabular}{lccc}
\hline & \multicolumn{1}{c}{$\Delta \bar{v}(p) / \Delta p$} & & $\Delta \gamma(p) / \Delta p$ \\
\cline { 2 - 2 } & $\mathrm{cm}^{-1} \mathrm{MPa}^{-1}$ & & $\mathrm{~cm}^{-1} \mathrm{MPa}^{-1}$ \\
\hline PMMA & $-0.17 \pm 0.01$ & & $0.09 \pm 0.01$ \\
PE & $-0.18 \pm 0.01$ & & $0.06 \pm 0.01$ \\
PS & $-0.22 \pm 0.01$ & & $0.12 \pm 0.01$ \\
\hline
\end{tabular}

lineshift. It is evident, that the method of hole burning, which uses only small pressure variations, is especially well suited for determining the linear shift parameters (see below). Figure 3 also shows that the lineshifts are accompanied by reversible line broadenings. The latter allow some insight into the local degree of order around the dye molecules and will be discussed below. The measured values for the shifts and broadenings are given in Table I.

\section{DISCUSSION OF THE PRESSURE EFFECTS}

In the literature quantitative phenomenological descriptions of spectral lineshifts $\Delta \bar{v}(p)$ of dye molecules in various host materials have been discussed by numerous authors (see for instance ref $13-15$ and references therein).

If one considers only small pressure variations, the results of the above mentioned theories can be linearized and one gets

$$
\Delta \bar{v}(p)=\Delta \bar{v}_{0}+C \kappa\left(p-p_{0}\right) \equiv \Delta \bar{v}_{0}+C \kappa \Delta p
$$

where $\kappa$ is the hydrostatic compressibility of the matrix and $C$ is a constant characterizing the coupling between the dye molecule and its matrix environment ( $C$ contains matrix elements with ground and excited state wavefunctions; see ref 13).

In the following we will show, how an equation equivalent to eq 2 can be obtained by considering the pressure effects as small perturbations in the solvent shift and by expanding the latter into a Taylor series. The starting point is eq 1 which can be rewritten as

$$
\Delta \bar{v}_{0}=K \cdot \bar{R}^{-6}
$$

by only considering the radial dependency. Such a relation has also been assumed by Drickamer recently. ${ }^{16} \mathrm{~K}$ is a constant containing molecular parameters of host and solute. Performing the expansion of eq 3 and by only carrying linear terms we obtain

$$
\Delta \bar{v}(p)=\Delta \bar{v}_{0}(1+2 \kappa \Delta p)
$$

where $\kappa$ is again the hydrostatic compressibility. Since we are dealing with extremely small pressure variations, we can consider the spectral shift as being linear in $p$ and, hence, use our experimental values of $\Delta p$ instead of $p$. We get by comparing eq 2 and 4

$$
C=2 \Delta \bar{v}_{0}
$$

Therefore the pressure shifts of the optical transitions depend in a linear fashion on the hydrostatic compressibility which is characteristic of the host material and the solvent shift describing the dye-matrix-interaction. With eq 4 it is possible to determine the compressibility of the matrices by measuring the pressure shifts $\Delta \bar{v} / \Delta p$ of the holes and by evaluating the solvent shift of the host-guest system. The latter can be obtained by comparing the gas phase spectra ${ }^{17,18}$ with the corresponding spectra in solid solution. ${ }^{19}$

Table II shows the solvent shifts, the compressibilities as obtained by our measurements and, for comparison, the compressibilities obtained by mechanical measurements. ${ }^{20}$ The correlation between the optically and mechanically measured values is surprisingly good, considering the crude assumptions which have been made in our calculation. Besides the pressure shifts the broadenings of the holes give additional information. The line broadenings reflect the dispersion in the shift parameters of the various molecules, which were 
Table II. Solvent shifts $\Delta \bar{v}_{0}$, compressibilities $\kappa_{\exp }$ as obtained by our optical experiments and mechanically measured low-temperature-compressibilities as taken from the literature $\kappa_{\text {lit }}$

\begin{tabular}{|c|c|c|c|}
\hline & $\Delta \bar{v}_{0}$ & $\kappa_{\exp }$ & $\kappa_{\text {lit }}$ \\
\hline & $\mathrm{cm}^{-1}$ & $\mathrm{GPa}^{-1}$ & $\mathrm{GPa}^{-1}$ \\
\hline PMMA & $-566 \pm 10$ & $0.150 \pm 0.028$ & 0.130 \\
\hline PE & $-690 \pm 10$ & $0.130 \pm 0.020$ & 0.141 \\
\hline PS & $-742 \pm 10$ & $0.148 \pm 0.021$ & 0.179 \\
\hline
\end{tabular}

originally selected at the laser frequency. The magnitude of the broadening is therefore a measure of the degree of order of the matrix: a perfect single crystal should only exhibit lineshifts without line broadening. On the other hand, the local degree of order is also reflected in the width of the inhomogeneous band. Taking this into account, the following equation should hold:

$$
\frac{\Gamma_{0}}{\Delta \bar{v}_{0}}=\frac{\Delta \gamma(p)}{\Delta \bar{v}(p)}=\frac{\Delta \gamma(p)}{\Delta p} \frac{\Delta p}{\Delta \bar{v}(p)}
$$

$\Gamma_{0}$ is the width of the inhomogeneous band, $\bar{v}_{0}$ is the solvent shift, $\Delta \gamma(p) / \Delta p$ is the broadening of the hole and $\Delta \bar{v}(p) / \Delta p$ is the shift of the hole. Table III gives the widths of the inhomogeneous bands $\Gamma_{0}$ and the two sets of parameters as given by eq 6 . A correlation seems to be reasonable, if one assumes, that both sets of data reflect the long range attractive part of the potentials determining both, the solvent shifts and the hole shifts with small pressure variations as applied in the hole burning experiment.

In Table $\mathrm{I}$ the broadenings in PE and PMMA are of the same magnitude, whereas the one in PS is larger due to the higher compressibility of PS. In Table III, on the other hand, the values for PMMA as PS are of the same order, whereas the value for PE is smaller. This shows, that eq 6 characterizes mainly the degree of order, as reflected in the widths of the inhomogeneous bands. Due to the partially crystalline structure of PE, the
Table III. Widths of the inhomogeneous bands $\Gamma_{0}$, widths devided by the solvent shifts $\Gamma_{0} / \Delta \bar{v}_{0}$ and pressure broadenings devided by the pressure shifts $\Delta \gamma(p) / \Delta \bar{v}(p)$

\begin{tabular}{lccc}
\hline & $\Gamma_{0}$ & & \\
& \multicolumn{1}{c}{$\mathrm{cm}^{-1}$} & $\Gamma_{0} / \Delta \bar{v}_{0}$ & $\Delta \gamma(p) / \Delta \bar{v}(p)$ \\
& & & \\
\hline PMMA & $276 \pm 10$ & $-0.53 \pm 0.04$ & $-0.53 \pm 0.07$ \\
PE & $276 \pm 10$ & $-0.18 \pm 0.03$ & $-0.31 \pm 0.06$ \\
PS & $116 \pm 10$ & $-0.41 \pm 0.03$ & $-0.55 \pm 0.05$ \\
\hline
\end{tabular}

inhomogeneous band is considerably narrower than the ones measured for PS and PMMA.

Since eq 6 holds, $\Delta \bar{v}(p)$ can be eliminated by using eq 4 and one gets a new equation for determining the compressibility from purely optical data.

$$
\Delta \gamma(p)=2 \Gamma_{0} \kappa \Delta p
$$

In conclusion it can be said, that the pressure tuning of homogeneous optical transitions is an extremely sensitive probe for the dye-matrix-interactions and also for the investigation of matrix parameters. In the above experiments the compressibilities could be measured without any mechanical contact to the sample. This opens a wide field for a variety of technical applications. Because of the local sensitivity of the hole burning method it is also appropriate for the detection of local strainfields and cracks in simple polymers and possibly in composite materials, where the detection of cracks and local strains is basically an unsolved problem.

\section{STARK EFFECT; BROADENING OF SPECTRAL HOLES}

In a similar way as strain fields, an externally applied static electric field can change the shape of a spectral hole. The energy levels of the dye molecules shift in the field due to multipolar interactions of the molecular charge distribution and because of polarization effects. In the case of a homogeneous 
field a dipole moment gives rise to an energy shift which is linear in field strength, while the shift due to polarization is quadratic to lowest order. ${ }^{21.22}$ The optical absorption line of a dye molecule or color center therefore shifts in the field, if the dipole moments or the polarizabilities are different in the two electronic states belonging to the observed transition. Because of its high resolution, the hole burning technique is capable of measuring very small line shifts caused by field strengths of a few $\mathrm{kV} \mathrm{cm}^{-121-27}$ In contrast to single crystals, where a hole usually splits into several components when a field is applied, ${ }^{23}$ in amorphous hosts the guest molecules are oriented randomly so that a hole broadens upon applying the field without being shifted. ${ }^{22,24} \mathrm{~A}$ reversible electricfield-induced hole filling was also observed to occur in the system perylene in poly(vinyl butyral), ${ }^{25}$ and it was shown to follow a linear field dependency. Since the free perylene molecule does, due to its inversion symmetry, not possess permanent dipole moments in its electronic ground or excited state, the authors of ref 25 explained their observation as being due to matrix-induced dipole moments. They assumed that among the dye molecules there is not one single value of the difference between the dipole moments in the two electronic states, but a distribution described by a Gaussian statistic, since each molecule 'sees' a different chemical environment.

In the following we briefly describe a simple phenomenological model which yields the shape of a spectral hole in an external electric field, when the absorbers are oriented in the matrix in a random fashion. We separately discuss the two cases that there is either one single value or a distribution of values of the difference $\Delta \mu$ between the dipole moments in the electronic ground and excited state of the dye molecules. We compare our theoretical results with experimental hole burning data, which were taken on the polymer poly(vinyl butyral) doped either with free-base chlorin or with a substituted $\mathrm{Zn}$-tetrabenzoporphin.

\section{THEORY}

First we assume that all the dye molecules in the sample are characterized by the same value $\Delta \mu$ of the dipole moment difference in the electronic ground and excited state. The vectors $\Delta \boldsymbol{\mu}$, however, are oriented at random. Then the absorption peak of each dye molecule is shifted linearly by the applied electric field $E$, according to the formula

$$
\Delta \omega_{\mathrm{E}}=-\frac{\Delta \mu \xi E}{\hbar} \cos v
$$

Here $\xi$ is the Lorentz correction factor which accounts for local electric field corrections in the condensed phase. It is given by $\xi=(\varepsilon+2) / 3$ with $\varepsilon$ being the dielectric constant of the sample. $v$ is the angle between the vector $\Delta \mu$ and the field vector $\boldsymbol{E}$. According to eq 8 , the line shift of the molecule in a constant electric field is determined solely by its orientation. Thus we can claculate the distribution function of those molecules which belong to a single site, i.e., which absorb at the same frequency at zero field. When the field is switched on, they are spread out to a rectangular distribution

$$
g_{\mathrm{E}}\left(\Delta \omega_{\mathrm{E}}\right)=\left\{\begin{array}{lll}
\frac{\hbar}{2 \Delta \mu \xi E} & \text { for } & \left|\Delta \omega_{\mathrm{E}}\right| \leqq \frac{\Delta \mu \xi E}{\hbar} \\
0 & \text { for } & \left|\Delta \omega_{\mathrm{E}}\right|>\frac{\Delta \mu \xi E}{\hbar}
\end{array}\right.
$$

The above function is normalized to one. A graphical representation is shown in Figure 5. The maximal frequency displacements of $\pm \Delta \mu \xi E / \hbar$ occur for those molecules whose $\Delta \mu$ vectors lie antiparallel and parallel to the $E$ field, respectively. The shifts of some other orientations are shown in Figure 5.

With the electric field switched off, the shape of a spectral hole is given by the convolution of the site distribution function $n z(\omega)$ of the photochemically converted molecules and the 


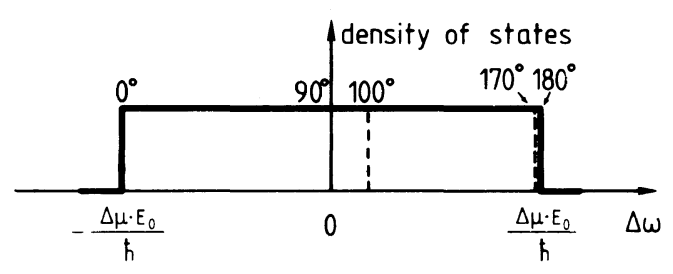

Step-Function-Distribution

Figure 5. Distribution function of the absorption frequencies of guest molecules in the electric field $E_{0}=$ $\xi E$, which belong to a single site in zero field. All molecules have the same dipole moment difference $\Delta \mu$, but are oriented in a random fashion with respect to the external $E$-field. The numbers give the orientation angles for some frequency shifts.

homogeneous absorption or fluorescence line $z(\omega)$ (see, for instance, ref 28 ).

$$
h_{0}(\omega)=(n z * z)(\omega)
$$

Both functions usually have Lorentzian shapes; the full halfwidth of $z(\omega)$ is $\gamma$ and the full halfwidth of $n z(\omega)$ is $\gamma_{D}$ with $\gamma_{D} \geqq \gamma$. In the electric field each single frequency within the site distribution function is spread out to a rectangular profile so that the resulting hole shape is given by the following equation.

$$
h_{\mathrm{E}}^{1}(\omega)=\left[\left(n z * g_{\mathrm{E}}\right) * z\right](\omega)
$$

The superscript 1 denotes that there is only one value of $\Delta \mu$ characterizing the absorbers. The two convolutions in eq $10 \mathrm{~b}$ can be carried out analytically; introducing dimensionless variables we get

$$
\begin{aligned}
& h_{f}^{1}(x)=(2 \pi f)^{-1} \\
& \left\{\begin{array}{lll}
\frac{\pi}{2} & \text { for } & x^{2}>f^{2}-1 \\
\pi+\arctan \left(\frac{2 f}{1-f^{2}+x^{2}}\right) & \text { for } & x^{2}=f^{2}-1 \\
1-f^{2}+x^{2} & \text { for } & x^{2}<f^{2}-1
\end{array}\right.
\end{aligned}
$$

The new variables are defined in the following way: $f:=2 \Delta \mu \xi E / \hbar\left(\gamma_{\mathrm{D}}+\gamma\right)$ is the ratio between the width of the dipolar energy distribution in the electric field and the initial hole width (for $E=0$ ).

$x:=2\left(\omega-\omega_{L}\right) /\left(\gamma_{D}+\gamma\right)$ is the frequency displacement from burning frequency, devided by the initial hole halfwidth.

In the preceding calculations we had assumed that all the dye molecules in the sample have the same dipole moment difference $\Delta \mu$ between their electronic ground and excited state. This model is expected to be most realistic in the case of polar dye molecules, whose dipole moment is intrinsic and is determined by the molecular structure. In order to explain the observed linear Stark effect on poly(vinyl butyral) doped with the unpolar dye perylene, Bogner et al. ${ }^{25}$ assumed that dipole moments are induced in the guest molecules by the polar matrix. In this case, however, due to the fluctuating surroundings of the molecules, a distribution of $\Delta \mu$ values rather than a single value is to be expected. The authors of ref 25 used a Gaussian statistic as given by eq 12 to describe their experiments.

$$
g(\Delta \mu)=\frac{4}{\pi^{1 / 2}(\overline{\Delta \mu})^{3}}(\Delta \mu)^{2} \exp \left[-\left(\frac{\Delta \mu}{\overline{\Delta \mu}}\right)^{2}\right]
$$

$\overline{\Delta \mu}$ is the most probable value of the $\Delta \mu$ distribution. We can calculate the hole shape for the case of a distribution of $\Delta \mu$ values by a superposition of shape functions as given by eq 11 which belong to different $\Delta \mu$ values and, hence, to different $f$ values:

$$
\begin{aligned}
h_{f}^{d}(x)= & \frac{2}{\pi^{3 / 2} \bar{f}^{3}} \\
& \times\left\{\int_{0}^{\sqrt{1+x^{2}}} f \exp \left[-\left(\frac{f}{\bar{f}}\right)^{2}\right] \arctan \right. \\
& \times\left(\frac{2 f}{1-f^{2}+x^{2}}\right) \mathrm{d} f \\
& +\int_{\sqrt{1+x^{2}}}^{\infty} \exp \left[-\left(\frac{f}{\bar{f}}\right)^{2}\right][\pi+\arctan \\
& \left.\left.\times\left(\frac{2 f}{1-f^{2}+x^{2}}\right)\right] \mathrm{d} f\right\}
\end{aligned}
$$




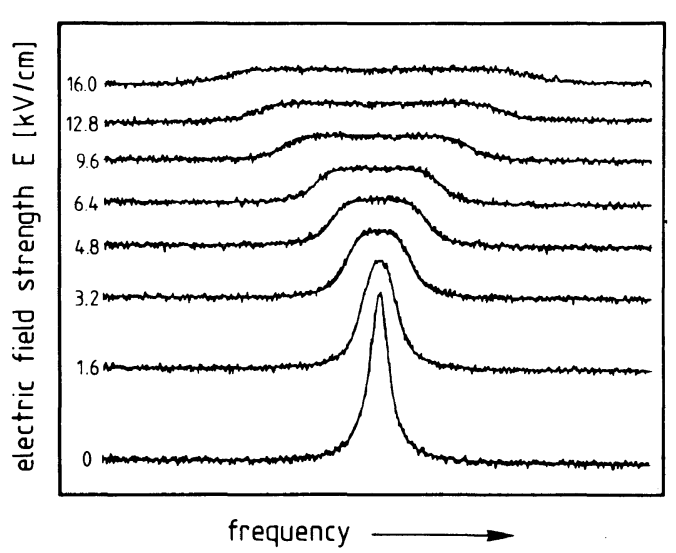

Figure 6. Measured hole profiles in the system PVB/ $\mathrm{H}_{2} \mathrm{Ch}$ at various electric field strengths. The initial hole halfwidth is $431 \mathrm{MHz}$. The smooth curves are theoretical and have been calculated with eq 11; their heights have been corrected for a back-reaction of the photoproduct.

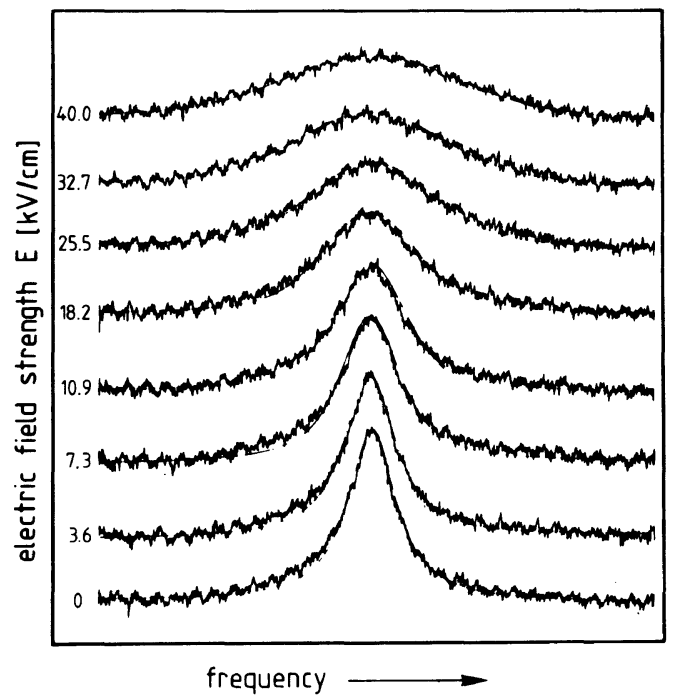

Figure 7. Measured hole profiles in the system PVB/ $\mathrm{Zn}-\mathrm{TBP}\left(\mathrm{C}_{6} \mathrm{H}_{4} \mathrm{CH}_{3}\right)_{4}$ at various electric field strengths. The initial hole halfwidth is $3.17 \mathrm{GHz}$. The smooth curves are theoretical and have been calculated with eq 13; their heights have not been corrected. $f$ :

Here the parameter $\bar{f}$ is defined in analogy to $\bar{f}:=2 \overline{\Delta \mu} \xi E / \hbar\left(\gamma_{\mathrm{D}}+\gamma\right)$, and the superscript $d$ denotes that a distribution of $\Delta \mu$ values has been used. The integrations in eq 13 must be performed numerically. Plots of the func- tions as given by eq 11 and 13 together with measured hole profiles are shown in Figures 6 and 7 , respectively.

\section{COMPARISON WITH EXPERIMENT}

In order to test our theoretical hole shape calculations we performed optical hold burning experiments on thin films of poly(vinyl butyral) (PVB) which were doped with the following two porphyrine dyes. As a slightly polar absorber we used free-base chlorin $\left(\mathrm{H}_{2} \mathrm{Ch}\right)$, as an unpolar absorber a symmetrically substituted $\mathrm{Zn}$-tetrabenzoporphin ( $\mathrm{Zn}$ TBP $\left.\left(\mathrm{C}_{6} \mathrm{H}_{4} \mathrm{CH}_{3}\right)_{4}\right)$. Structural formulae of the guest molecules are shown in Figure 2 . Burning and scanning of the holes were performed with a tunable single-mode $c w$ dye laser at a temperature of $1.95 \mathrm{~K}$. In each case the holes were burnt into the lowest-energy absorption bands of the dye molecules. For applying an electric field the sample films were placed between two glass plates whose inner surfaces were coated with thin electrically conducting but optically transparent $\mathrm{SnO}_{2}$ layers. The field strengths applied to the samples were in the range up to $40 \mathrm{kV} \mathrm{cm}^{-1}$. In Figures 6 and 7 we have plotted the measured hole profiles for the case of the polar and the unpolar dopant molecules, respectively. In each case the recorder traces correspond to subsequent scans over the same hole, taken at different electric field strengths. Additionally, in the figures the theoretical hole shape curves have been plotted as calculated by using eq 11 (Figure 6) and eq 13 (Figure 7). Especially in the case of the chlorin sample (Figure 6) the theoretical curves are hardly distinguishable from the measured hole profiles. As can be seen from the figures, the hole shape behaves quite differently in the electric field when the matrix is doped with polar and unpolar dye molecules. In the case of the polar molecule chlorin it looses its bell shape and becomes flat in its central region, thereby reflecting the convo- 
lution of the Lorentzian curve with a rectangle.

From the comparison of the measured profiles with the theoretical curves we can calculate a dipole moment difference $\Delta \mu$ of $(0.214 \pm 0.011) \mathrm{D}$ for chlorin. For the dielectric constant we used the value of $\varepsilon=3.0 .^{22}$ The $\Delta \mu$ value has been obtained from the increase of the measured hole widths. For long experimental observation periods a back-reaction of the photoproduct slightly diminishes the area and, thus, the depth of the hole. Therefore, the heights of the fit curves in Figure 6 have in each case been reduced in a way in which optimal agreement with the measured profiles was obtained. Although our experimental and theoretical hole shapes agree quite well, there are two features of the experimental profiles which are not accounted for by the theory. The first one is a slight indentation at the center of the hole, which shows up at higher field strengths. This dip, which causes a double-maximum structure, is due to the fact that in the burning and in the probing process those dye molecules whose electric transition dipole moments lie parallel to the polarization direction of the laser, are weighted stronger than other orientations, thus causing a dichroic character of the hole. ${ }^{28,29}$ Since in a molecular coordinate system the difference vector $\Delta \boldsymbol{\mu}$ between the static dipole moments and the transition dipole are oriented under a fixed angle of $90^{\circ},{ }^{27}$ an isotropic distribution of the orientations of the $\Delta \boldsymbol{\mu}$ vectors in the sample, as assumed for the theoretical calculations, does not exist. A second discrepancy between theory and experiment is caused by the fact that in the dye molecules not exactly one single $\Delta \mu$ value exists. Besides the intrinsic dipole moment difference characteristic of the free chlorin molecule, additional dipole moments are induced by the polar matrix which differ from molecule to molecule in both direction and size. The resulting distribution of $\Delta \mu$ values tends to flatten the hole wings. Both effects, however, cause only minor deviations of the measured hole shapes from the theoretical curves. Our simple analytical theory seems to adequately describe the main features of the broadening of spectral holes for the case of polar dye molecules.

The measured hole shapes of the unpolar porphyrine dye $\mathrm{Zn}-\mathrm{TBP}\left(\mathrm{C}_{6} \mathrm{H}_{4} \mathrm{CH}_{3}\right)_{4}$ together with the theoretical fits are shown in Figure 7. The hole clearly behaves quite differently from the case of the chlorin sample, when the electric field is raised. It retains its bell shape up to the highest field strengths applied and does not adopt a rectangular shape. There is also no tendency to form a double-maximum structure; in all scans the point of minimal absorption (bottom of the hole) is at the burning frequency. The bell shape shows that there is not one single rectangular energy distribution of the molecular dipole moment differences, but rather a distribution of rectangles. With the assumption of a Gaussian distribution of the $\Delta \mu$ values we can fit the measured profiles quite well. The theoretical curves plotted in Figure 7 have been calculated using a mean induced dipole moment different $\overline{\Delta \mu}$ of $(0.174 \pm 0.015) \mathrm{D}$, which was obtained as a mean value from width and depth fits. For this sample, the hole area does not decrease. with time; as a consequence, the theoretical fit curves have not been corrected in height.

Figure 7 shows that the model of a statistical distribution of $\Delta \mu$ values yields good agreement between experimental and theoretical hole shapes in the case of unpolar dye molecules. The origin of the dipole moments is, most likely, the electric crystal field or 'reaction field' in the matrix. Because of the different chemical 'micro-environments' of the various molecules of the ensemble, the assumption of a statistical distribution of these intrinsic field strengths seems quite reasonable. If we take for the difference of the polarizabilities of the aromatic dye molecules in their groud and excited electronic state a typical value of $\Delta \alpha=50 \AA^{330}$ (neglecting the tensor character) we can estimate the order of magnitude of the crystal field; for its most 
probable value we get $\overline{E_{\text {int. }}}=1.3 \times 10^{7} \mathrm{~V} \mathrm{~cm}^{-1}$. Although this value may be uncertain within a factor of two to three the method of applying an external electric field to spectral holes provides, for the first time, the possibility of measuring internal fields in polymers.

Acknowledgements. We would like to thank our colleagues J. Friedrich and A. Blumen for many helpful discussions. Part of this work was supported by a grant of the Stiftung Volkswagenwerk.

\section{REFERENCES}

1. B. M. Kharlamov, R. I. Personov, and L. A. Bykovskaya, Opt. Comm., 12, 191 (1974).

2. A. A. Gorokhovskii, R. K. Kaarli, and L. A. Rebane, JETP Lett., 20, 216 (1974).

3. L. A. Rebane, A. A. Gorokhovskii, and J. V. Kikas, Appl. Phys., B29, 235 (1982).

4. G. J. Small, "Spectroscopy and Excitation Dynamics of Condensed Molecular Systems," V. M. Agranovich and R. M. Hochstrasser, Ed., NorthHolland, Amsterdam, 1983, p 515.

5. J. Friedrich and D. Haarer, Angew. Chem. Int. Ed. Engl., 23, 113 (1984).

6. K. S. Schweizer and D. Chandler, J. Chem. Phys., 76, 2296 (1982).

7. H. C. Henke, W. Yu, H. L. Selzle, E. W. Schlag, D. Wutz, and S. H. Lin, Chem. Phys., 97, 205 (1985).

8. H. C. Longuet-Higgins and J. A. Pople, J. Chem. Phys., 27, 192 (1957).

9. P. C. Johnson and H. W. Offen, J. Chem. Phys., 57, 336 (1972).

10. W. W. Robertson, J. Chem. Phys., 33, 362 (1961).

11. T. G. Politis and H. G. Drickamer, J. Chem. Phys., 74, 263 (1981).

12. W. Richter, G. Schulte, and D. Haarer, Opt. Commun., 51, 412 (1984).

13. S. H. Lin, J. Chem. Phys., 59, 4458 (1973).
14. D. Curie, D. E. Berry, and F. Williams, Phys. Rev., B20, 2323 (1979).

15. C. P. Slichter and H. G. Drickamer, Phys. Rev., B22, 4097 (1980).

16. F. F. Clark and H. G. Drickamer, J. Phys. Chem., 90, 589 (1986).

17. P. S. H. Fitch, L. Wharton, and D. H. Levy, J. Chem. Phys., 69, 3424 (1978).

18. P. S. H. Fitch, L. Wharton, and D. H. Levy, J. Chem. Phys., 70, 2018 (1979).

19. G. Schulte, Ph. D. thesis, University of Bayreuth (1986).

20. I. Perepechko, Ed., "Low-Temperature Properties of Polymers," Moscow/Oxford, 1980.

21. V. D. Samoilenko, N. V. Rasumova, and R. I. Personov, Opt. Spectrosc. (USSR), 52, 346 (1982).

22. F. A. Burkhalter, G. W. Suter, U. P. Wild, V. D. Samoilenko, N. V. Rasumova, and R. I. Personov, Chem. Phys. Lett., 94, 483 (1983).

23. A. I. M. Dicker, L. W. Johnson, M. Noort, and J. H. van der Waals, Chem. Phys. Lett., 94, 14 (1983).

24. A. P. Marchetti, M. Scozzafava, and R. H. Young, Chem. Phys. Lett., 51, 424 (1977).

25. U. Bogner, P. Schätz, and M. Maier, Chem. Phys. Lett., 102, 267 (1983).

26. U. P. Wild, A. Renn, A. J. Meixner, and S. E. Bucher, 2nd International Conference on Unconventional Photoactive Solids, Cleveland, Ohio, 1985, p 19.

27. A. J. Meixner, A. Renn, S. E. Bucher, and U. P. Wild, XIth Molecular Crystal Symposium, Lugano, Switzerland, 1985, p 198.

28. L. Kador, G. Schulte, and D. Haarer, J. Phys. Chem., 90, 1264 (1986).

29. W. Köhler, W. Breinl, and J. Friedrich, J. Phys. Chem., 89, 2473 (1985).

30. J. H. Meyling, W. H. Hesselink, and D. A. Wiersma, Chem. Phys., 17, 353 (1976); measured for the components of the $\Delta \alpha$ tensor of tetracene the values $29 \AA^{3}, 25 \AA^{3}$, and $5 \AA^{3}$ and for pentacene $53 \AA^{3}$, $90 \AA^{3}$, and $-11 \AA^{3}$, respectively. We assume that the $\Delta \alpha$ values of porphyrine dyes should be of a comparable magnitude. 